\title{
Performance Evaluation of 802.11p-Based Ad Hoc Vehicle-to-Vehicle Communications for Usual Applications Under Realistic Urban Mobility
}

\author{
Patrick Sondi ${ }^{1}$, Martine Wahl ${ }^{2}$, Lucas Rivoirard ${ }^{3}$, Ouafae Cohin ${ }^{4}$ \\ ${ }^{1}$ ULCO, LISIC, EA 4491, F-62228 Calais, France \\ ${ }^{2,3}$ IFSTTAR, COSYS, LEOST, F-59650 Villeneuve d'Ascq, France \\ ${ }^{1,2}$ Univ Lille Nord de France, F-59000 Lille, France \\ ${ }^{4}$ Hautes Etudes d'Ingénieur (HEI), F-59000 Lille, France
}

\begin{abstract}
In vehicular ad hoc networks, participating vehicles organize themselves in order to support lots of emerging applications. While network infrastructure can be dimensioned correctly in order to provide quality of service support to both vehicle-to-vehicle and vehicle-to-infrastructure communications, there are still many issues to achieve the same performance using only ad hoc vehicle-to-vehicle communications. This paper investigates the performance of such communications for complete applications including their specific packet size, packet acknowledgement mechanisms and quality of service requirements. The simulation experiments are performed using Riverbed (OPNET) Modeler on a network topology made of 50 nodes equipped with IEEE 802.11p technology and following realistic trajectories in the streets of Paris at authorized speeds. The results show that almost all application types are well supported, provided that the source and the destination have a direct link. Particularly, it is pointed out that introducing supplementary hops in a communication has more effects on endto-end delay and loss rate rather than mobility of the nodes. The study also shows that ad hoc reactive routing protocols degrade performance by increasing the delays while proactive ones introduce the same counter performance by increasing the network load with routing traffic. Whatever the routing protocol adopted, the best performance is obtained only while small groups of nodes communicate using at most two-hop routes.
\end{abstract}

Keywords-V2V; 802.11p; QoS; Urban mobility; Simulation

\section{INTRODUCTION}

Vehicular ad hoc networking is an emerging paradigm where participating vehicles can exchange directly various information such as warnings, traffic conditions, and many other data. While network infrastructure can be dimensioned correctly in order to provide quality of service (QoS) support to both vehicle-to-vehicle (V2V) and vehicle-to-infrastructure (V2I) communications, there are still many issues to achieve the same performance using only ad hoc vehicle-to-vehicle communications. For several reasons such as the absence of infrastructure in some areas, its destruction after an accident or a disaster, or simply by opportunism, it may become necessary to rely only on ad hoc $\mathrm{V} 2 \mathrm{~V}$ communications in order to keep on providing the same services to vehicles. Performance of $\mathrm{V} 2 \mathrm{~V}$ communications is most evaluated at the level of wireless LAN based on differentiated traffic, thus regarding the characteristics of the data link and physical layers. However, due to the plethora of applications that are now available to the drivers through their smartphones connected to mobile communication technologies such as $3 \mathrm{G} / 4 \mathrm{G}$, V2V communications will not be developed further if the underlying technologies do not demonstrate their ability to support the same kind of applications. This work investigates the performance that can be expected from such communications, not only for differentiated traffic, but also for complete applications including their specific packet size, packet acknowledgement mechanisms, and QoS requirements. The main objective is to determine the performance that can be achieved with one of the technologies envisioned as a standard for V2V/V2I communications, namely IEEE 802.11p WAVE (Wireless Access for Vehicular Environments). For example: what performance a vehicle should expect when using a specific application ? Up to how many hops could be the vehicles sharing the application while keep a good QoS ? How many different traffic flows, of different types of service, can be involved in the same area simultaneously without degrading the performance for each application ? Particularly focused on real-world applications of vehicular ad hoc networks, this work targets evaluation of usual applications using realistic topology, mobility models, and network size while using standardized both ad hoc routing protocols and wireless LAN technologies.

The content of this paper is organized as follows. First, a related work about vehicular ad hoc networking and application is presented. Then, the system designed in order to perform simulation evaluation, including network topology, mobility models and simulation scenarios are described. Finally, the simulation results and performance analysis are reported and discussed, before the conclusion and prospective work are presented.

\section{RELATED WORK}

This work has been firstly motivated by the key-role that vehicle-to-vehicle $(\mathrm{V} 2 \mathrm{~V})$ communications will play in transportation and communication infrastructures with the growing penetration of electric vehicles [1] and the emergence of autonomous vehicle concept. On one hand, efficient resource management and service access could be achieved through vehicular cloud networks, and on the other hand, the passengers inside the vehicles could benefit of innovative applications while the vehicle will be in an almost autonomous 
driving mode most of the time. Several evaluations have been performed on V2V communications based on IEEE 802.11p $[2][3][4][5][6]$. The one presented in [2] is one of the most complete. Despite the quality of the investigations presented about the functioning of the data link and physical layers of this technology, this study does not allow catching 802.11p performance for concrete usual applications interacting with the users; that was not in the scope of the study. Thus, the second motivation of this work is to complete this part of the study of IEEE 802.11p-based vehicle-to-vehicle communication performance for usual applications. In absence of infrastructure, multi-hop V2V communications are dependent of ad hoc routing protocols: this is the only way to achieve the network management functions in a distributed manner. Despite the numerous proposals for vehicular ad hoc network routing protocols [7][8][9], the main routing protocols that are currently proceeding in the standardization process are those proposed for mobile ad hoc networks (MANETs) such as Dynamic Source Routing (DSR) [10], Ad hoc On-Demand Distance Vector (AODV) [11], Optimized Link State Routing Protocol (OLSR) [12], Topology Dissemination Based on Reverse-Path Forwarding (TBRPF) [13], and Open Shortest Path First-Overlapping Relay (OSPF-OR) [14]. It is interesting to evaluate how one protocol of each family of ad hoc routing protocols, namely reactive protocols such as AODV and proactive ones such as OLSR, affects the performance of V2V communications over IEEE 802.11p. Since they are all best effort routing protocols, it seems also interesting to evaluate some of their variants that provide an extension for quality of service (QoS) support. Quality of Service for Ad hoc Optimized Link State Routing Protocol (QOLSR) [15] is a variant of OLSR widely evaluated on applications with QoS requirements, but not yet with $802.11 \mathrm{p}$. Its complete specifications are presented in [16], and its implementation is available in OPNET contributed models.

\section{SYSTEM DESCRIPTION}

In a classical vehicular network infrastructure [17], Road Side Units (RSUs) are deployed along the roads and the streets in order to ensure a good coverage of the area where vehicles may request different services. Depending on the application, the communications between vehicles can transit through the RSUs or in ad hoc mode by multi-hop relaying from vehicle to vehicle. In this work, only this latter mode is evaluated due to the reasons previously mentioned in section 1 .

\section{A. Network topology}

Intuitively, referring to the results of different researches presented in section 2, notably [2], [17] and [15], the area that may be covered efficiently in fully ad hoc mode has a limited size. Thus, the network topology considered in this study covers approximately an area of $5 \mathrm{~km} \times 3 \mathrm{~km}$ dimensions.

Considering a sensing range of $1 \mathrm{~km}$, such an area allows reaching up to 5 hops on a straight line, which is sufficient for our study. In order to obtain accurate network density and diversity, the simulation is performed on an urban environment (Fig. 1) where the intersections of streets and roads allow avoiding particular cases such as isolated single roads or highways. The network is made of 50 nodes departed uniformly on the different routes in the streets of Paris. The same network sizes are used in similar evaluations [18], [19].

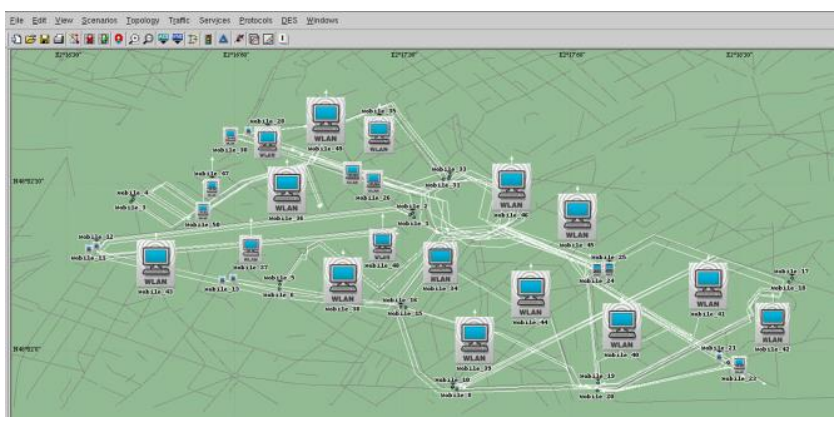

Fig. 1. Network topology for simulation

\section{B. Node mobility}

The nodes follow realistic trajectories defined along existing streets in Paris (Fig. 2). Up to 20 trajectories have been defined, each one followed by a pair of nodes initialized at different start point and time. Consequently, there are 10 fixed nodes departed over the area simulating stopped vehicles and $80 \%$ of mobile nodes in the network as in [18]. Each trajectory contains some steps where the node moves at $50 \mathrm{~km} / \mathrm{h}$ such as in long straight line, other where it moves at $30 \mathrm{~km} / \mathrm{h}$ such as when approaching intersections and $10 \mathrm{~km} / \mathrm{h}$ when turning around curves.

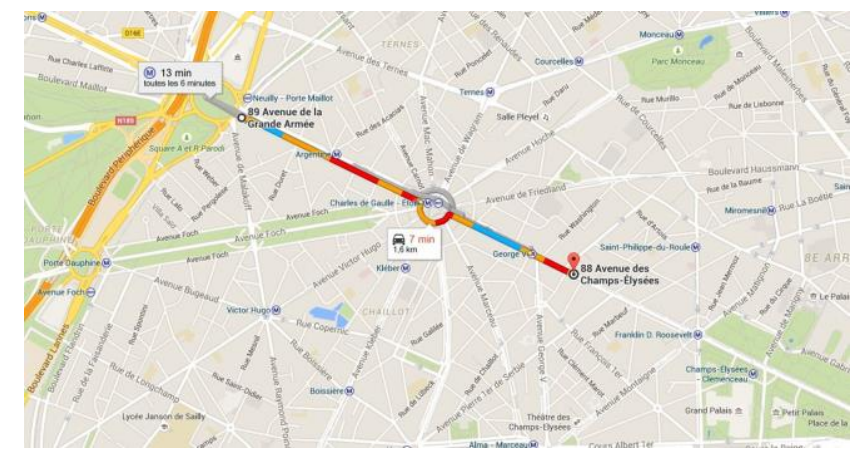

Fig. 2. Trajectory of node 28 on a real-world map (using Google maps)

In all the scenarios, each trajectory has a duration of about 6 minutes in one direction, then the node moves backward after a pausing time of 1 minute (Fig. 3). Some trajectories cover the same part of one street, in the same or in the opposite movement direction. It can be noticed that the trajectory of node 28 has the same duration in the simulation model (Fig. 3) as in a real-world itinerary (Fig. 2).

\section{Node configuration}

The nodes are equipped with IEEE 802.11p WAVE technology. As early proposed in [18], the transmit power is set at $0.02 \mathrm{~W}$ and receiver sensitivity at $-95 \mathrm{dBm}$ in order to obtain a communication range of $1 \mathrm{~km}$. These values were proposed before the model of IEEE 802.11p was available in Riverbed (OPNET) Modeler, and the evaluations carried out in this work confirm that they also work correctly in the official model now available. The wireless LAN configuration applied to the nodes is summarized in Fig. 4. 


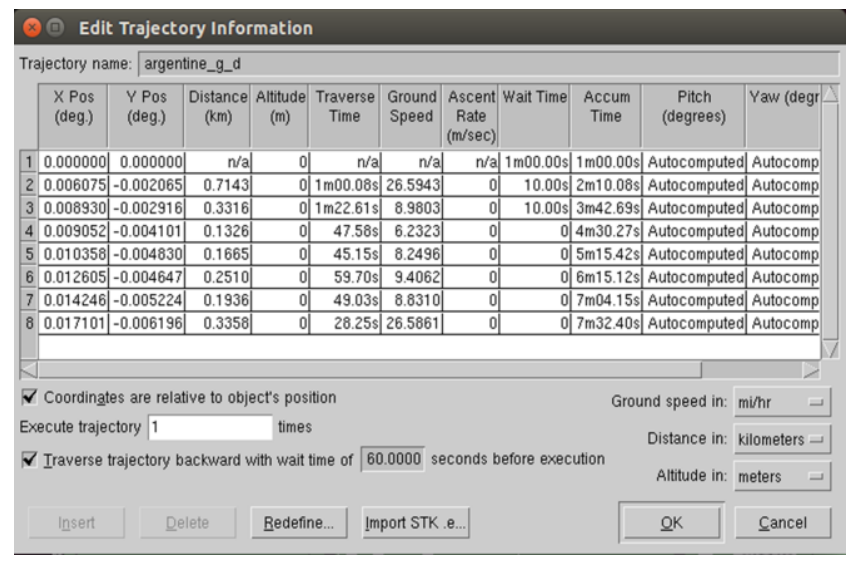

Fig. 3. OPNET model of trajectory of node 28 during simulation

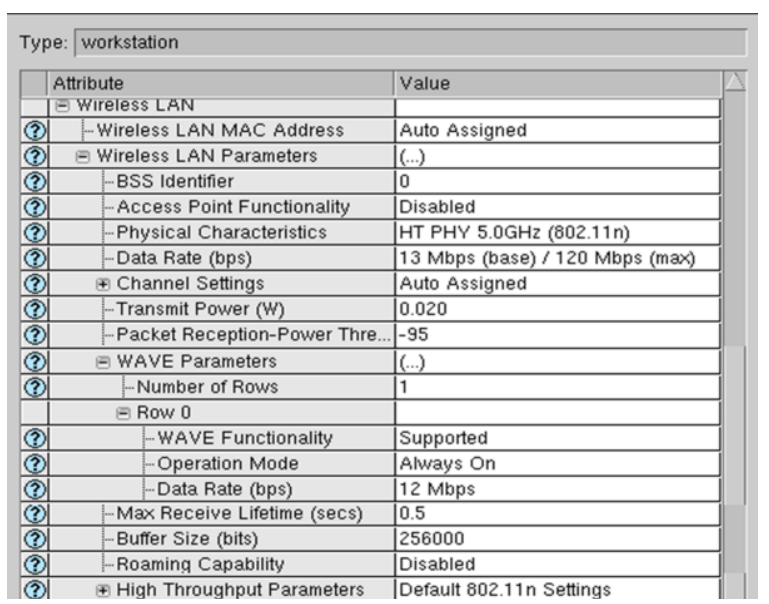

Fig. 4. Wireless LAN Parameters applied to the nodes

\section{Ad hoc routing protocols}

As mentioned in section 2, many routing protocols and their variants have been proposed especially for ad hoc vehicleto-vehicle communications. However, many recent works still consider original standardized ad hoc routing protocols when evaluating the performance of V2V communications [20]. In this work, the main objective is to evaluate the performance that the users could expect for usual applications over IEEE $802.11 \mathrm{p}$ vehicle-to-vehicle communications independently from the routing protocol. Since an ad hoc routing protocol is mandatory to operate ad hoc vehicle-to-vehicle communications, AODV and OLSR, respectively one reactive and one proactive ad hoc routing protocols, are used in order to study the advantages and drawbacks of each family of ad hoc routing protocols on performance. Some of the applications evaluated have quality of service $(\mathrm{QoS})$ requirements, but AODV and OLSR are best effort routing protocols that do not provide QoS support. In order to complete the study, two variants of OLSR with QoS support are also evaluate, namely:

- QOLSR : this routing protocol has been proposed in [15][16] as an extension of OLSR. The main idea is that each node uses the traffic received from the others in order to estimate locally the value of the metrics such as bandwidth, delay and loss on the route from each of them. This estimation is updated periodically and broadcasted through HELLO and TC messages so that all the nodes can take them into account when performing multipoint relay (MPR) selection and route computation to reach the originating node. It has been shown in [15] that QOLSR performs better than OLSR in large scale and congested ad hoc networks, particularly by maintaining a higher delivery ratio of the traffic from applications with QoS requirements such as voice and videoconference. However, the additional routing traffic introduced for QoS signaling increases the load on the WLAN thus consuming part of the resources that will lack to the applications. Previously mentioned work [15][16] realized the evaluation of QOLSR over 802.11g-based ad hoc networks and only with fixed nodes. In this work, the evaluations will be performed over $802.11 \mathrm{p}$ while considering realistic mobility of the vehicles.

- C231 : in order to avoid additional routing traffic introduced by QOLSR, another variant of OLSR that uses a single QoS metric instead of three is evaluated. In this variant, each node uses the location information of its neighbors in order to compute the expected received power from each neighbor and uses this information as a QoS metric. Since the network changes due to mobility, each node periodically updates the value of this metric and broadcasts it through HELLO and TC messages. The first assumption considered in this variant is that all the vehicles use the same transmit power value. The second one is that it is possible to compute accurately the value of the received power using an appropriate path loss propagation model. This latter subject has been an active area of research in recent years. Path loss arises when an electromagnetic wave propagates through space from transmitter to receiver. The power of the signal is reduced due to path distance, reflection, diffraction, scattering, free-space loss and absorption by the objects in the environment. It is also influenced by the different environment (i.e. urban, suburban and rural). The variations of the embedded transmitter and receiver antenna heights also produce losses. The losses present in a signal during propagation from transmitter to receiver may be classical and already existing. COST-231 WalfischIkegami model is an extension of COST Hata model and has been proposed for urban areas [21], [22]. It can be used for frequencies above $2000 \mathrm{MHz}$ when there is Line Of Site (LOS) between the transmitter node and the receiver node. It also takes into account various parameters such as the characteristics of buildings, roads and other obstacles which are important for a relevant prediction especially in urban areas. According to visibility conditions, only the case in LOS path loss calculation [23], [24] has been implemented. In this situation, there is no obstruction in the direct path between the transmitter and the receiver, and the estimated received power in $\mathrm{dB}$ is obtained using the equation (1):

$L_{L O S}(d B)=42,26+26 \log _{10}(d)+20 \log _{10}(f)$ 


\section{Where}

$f$ : Frequency of operation in $\mathrm{MHz}(5885 \mathrm{MHz}$ in $802.11 \mathrm{p}$ standard which is greater than $2000 \mathrm{MHz}$ )

\section{$\mathrm{d}$ : distance from the transmitter in kilometer}

This equation is only accurate in the far-field where spherical spreading can be assumed. It is not applicable when the receiver is close to the transmitter. In this study, the nodes are spaced of 300 meters in average

\section{E. Applications used by the vehicles}

The variety of applications that can be deployed on a vehicular cloud with their specific constraints is very large. In this work, four types of applications have been modeled to which any other application could be attached, at least based on an approximation of its functioning. These applications are:

- App_1 an application generating broadcast traffic : the source sends one packet of 800 bytes every 25 milliseconds (40 packets/second) to the entire network with type of service (TOS) set to best effort;

- App_2 a safety application generating unicast traffic : the source sends one packet of 800 bytes every 25 milliseconds (40 packets/second) to a specific destination with TOS set to delay and reliability;

- App_3 a voice application : the source calls a specific destination and they start a voice session of GSM quality level;

- App_4 a videoconferencing application : the source calls a specific destination and they start a videoconferencing session (configuration in Fig. 5);

() (Video Conferencing) Table
\begin{tabular}{|l|l|}
\hline Attribute & Value \\
\hline Frame Interarrival Time Information & 10 frames/sec \\
\hline Frame Size Information (bytes) & $128 \times 120$ pixels \\
\hline Symbolic Destination Name & Video Destination \\
\hline Type of Service & Interactive Multimedia (5) \\
\hline RSVP Parameters & None \\
\hline Traffic Mix $(\%)$ & All Discrete \\
\hline
\end{tabular}

Fig. 5. Videoconferencing application configuration

\section{SIMULATION RESUltS}

The Riverbed (OPNET) Modeler is used as the modeling and simulation environment for all the evaluations realized in this work. A model of IEEE 802.11p is provided with the Modeler, and also process models of both AODV and OLSR routing protocols. A model of QOLSR [16] has been obtained in the contributed models of OPNET website from which was derived a model of $\mathrm{C} 231$. Each simulation session represents 12 minutes of the vehicular network functioning.

\section{A. Simulation scenarios description}

The simulation scenarios are designed in order to evaluate the performance of the applications in the context of the vehicular network described in section 3. Particularly, the behavior of both the application and the wireless LAN are studied when each routing protocol operates, and the key points that determine the performance are analyze. A description of each scenario follows:

- Scenario_1: in this scenario, node 26 (see Fig. 1) is the source of App_1 described in section III.E and it broadcasts packets through the entire network. The objective is to analyze how this traffic reaches one-hop, two-hop and, if any, farther neighbors;

- Scenario_2: in this scenario, each node in the vehicular network is a source of App_1 and broadcasts packets through the entire network. The objective is to analyze how both the wireless LAN and each routing protocol react to a great amount of generated traffic;

- Scenario_3: in this scenario, a node is the source of App_2 and sends packets to a destination located onehop away. The objective is to evaluate the performance of a unicast traffic imposing a type of service similar to those of safety applications;

- Scenario_4: this scenario is the same as scenario_3, except that the destination is picked up three hops away and then it comes closer to the source;

- Scenario_5: in this scenario, a node is the source of App_3 and calls a destination located one-hop away. The objective is to evaluate the performance of voice conversation using vehicle-to-vehicle communication in urban mobility conditions;

- Scenario_6: this scenario is the same as scenario_5, except that the destination is picked up three hops away and then it comes closer to the source;

- Scenario_7: in this scenario, a node is the source of App_4 and calls a destination located one-hop away. The objective is to evaluate the performance of videoconferencing using vehicle-to-vehicle communication in urban mobility conditions;

- Scenario_8: this scenario is the same as scenario_7, except that the destination is picked up three hops away and then it comes closer to the source;

- Scenario_9: in this scenario, three pairs in the network are respectively the source and the destination of App_2, App_3 and App_4. The objectives are to evaluate the performance of each type of application in presence of concurrent traffic of other types, and to verify how the differentiated traffic management functionality of $802.11 \mathrm{p}$ is efficient according to the performance observed at the application level.

\section{B. Result analysis}

Each scenario is run several times with different seed values for the random number generator in order to avoid that the related sequence favor a particular routing protocol. The results presented and commented in this section are the average value of all the runs of the same scenario for each protocol. Consequently, the following results have been collected over a hundred simulations.

\section{1) Results for scenario 1 : a single broadcast traffic}



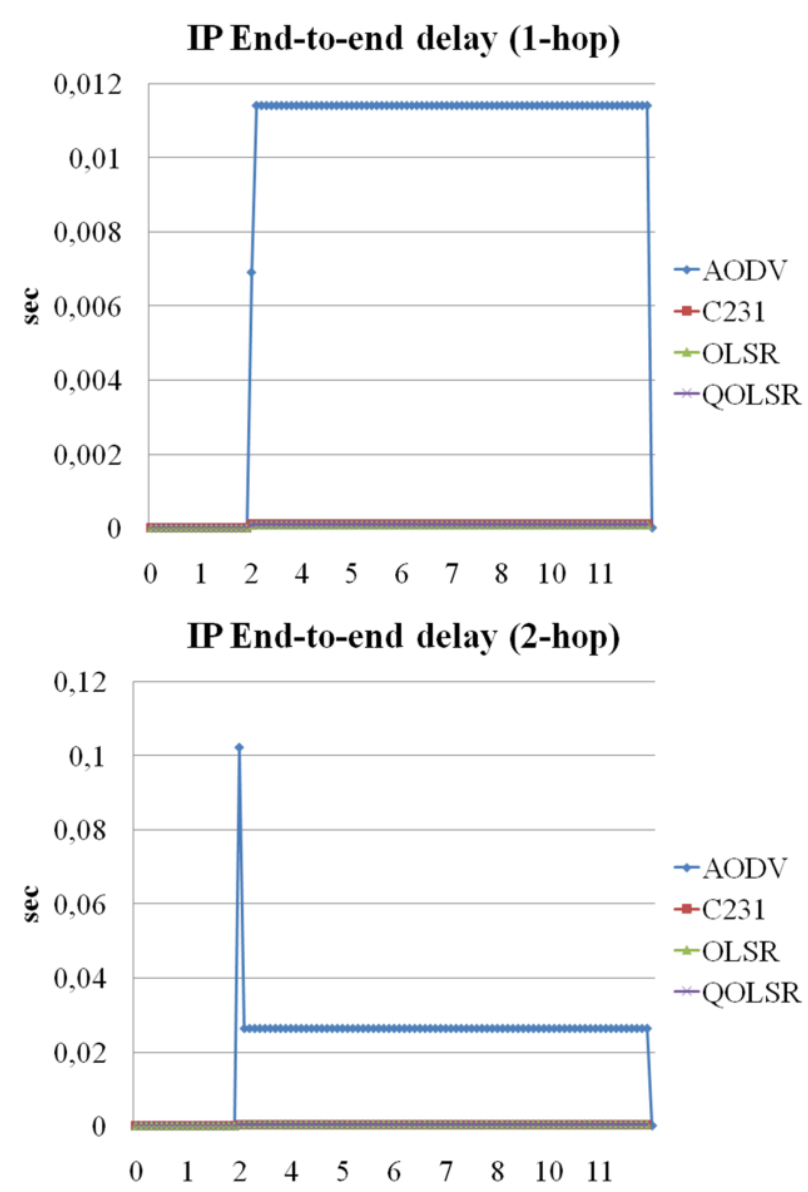

Fig. 6. End-to-end delay of broadcast traffic to 1-hop and 2-hop neighbors

In this scenario, two groups of nodes are obtained: the first is made of nodes that received the packets directly from the source (1-hop nodes), and the second that received them through a relaying node (2-hop nodes). Every nodes received correctly the packets (40 packets per second). AODV reaches the worst delay values in both cases, thus emphasizing its weaknesses in dealing with broadcast traffic. Taking into account that most applications involved in vehicular networks are broadcast-based, this is a critical issue about this protocol.

However, regarding the other protocols, the end-to-end delays achieved with $802.11 \mathrm{p}$ are lower than the milliseconds, thus ensuring very good performance for a safety application that requires a refresh time of $25 \mathrm{~ms}$.

2) Results for scenarios 3 and $4:$ a unicast application

The results presented in Fig. 7 show clearly that the application App_2 based on unicast traffic flow has good performance for a 1-hop destination. All 40 packets of 800 bytes sent per second are received with a low delay of $1.4 \mathrm{~ms}$. On Fig. 8, results for progressive hop count show that the delivery ratio of application traffic is less than $50 \%$ when the nodes are 3-hop away, it approaches $70 \%$ at 2-hop and then all the packets are received when the source and destination have direct link.
IP hop count
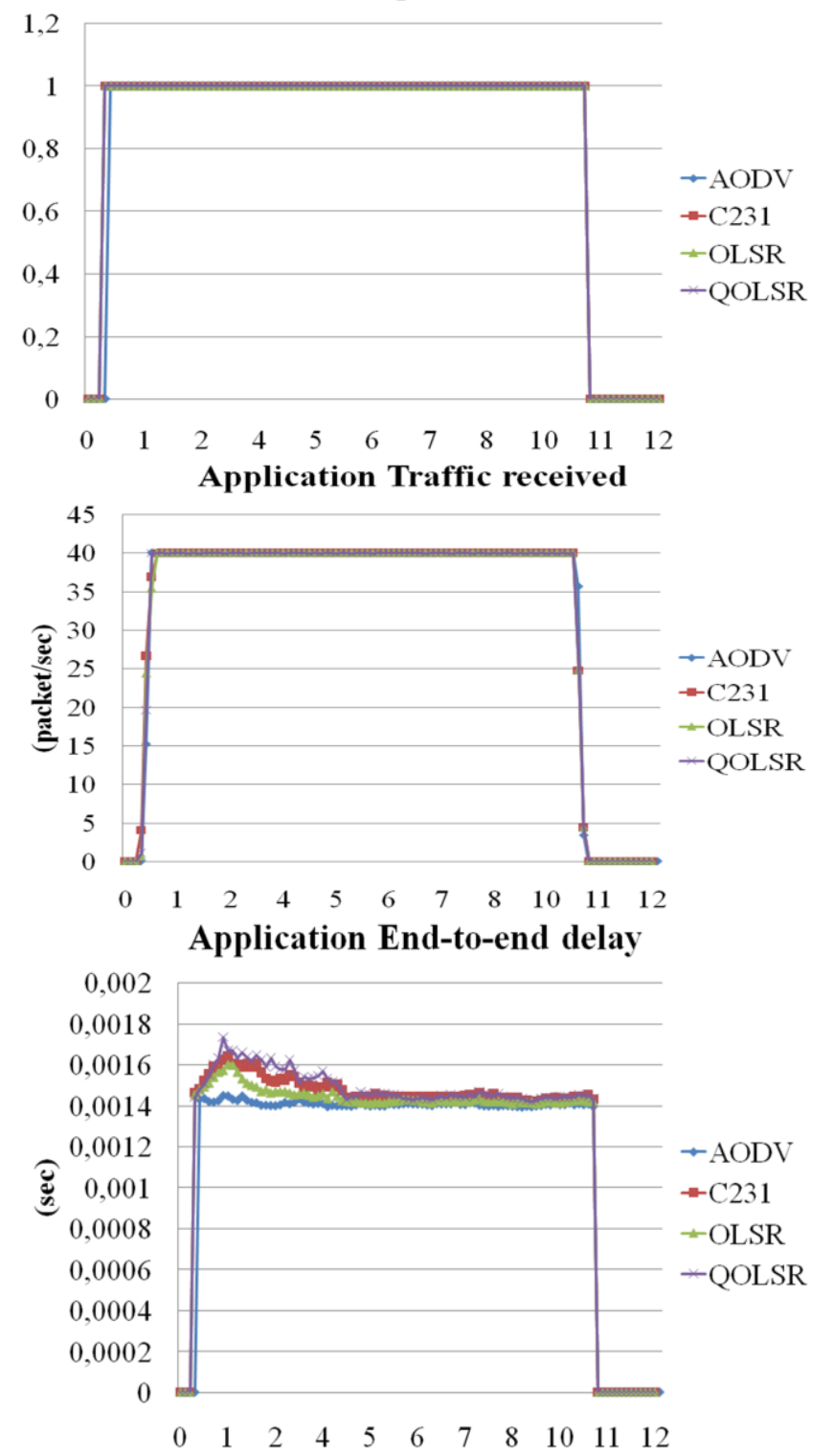

Fig. 7. Hop count, delivery, end-to-end delay for unicast traffic (scenario 3)

Many of the packets that were sent when the nodes were more than 1-hop away are not actually lost, but they are received with a delay. This explain why the destination receives up to 70 packets per second when only 40 were sent by the source. This evolution of the delivery ratio demonstrate that it is not suitable to target more than 2-hop destinations in V2V communication with $802.11 \mathrm{p}$, unless the application was neither loss-sensitive nor delay-sensitive. The best-effort routing protocols, AODV and OLSR, provide better performance than QoS variants which have the worst delays and delivery ratios. Due to the overhead introduced by QoS mechanisms, such solutions should not be used when only best-effort unicast traffic are involved. For such unicast besteffort traffic flow, AODV is clearly the best routing protocol. 


\section{IP hop count}

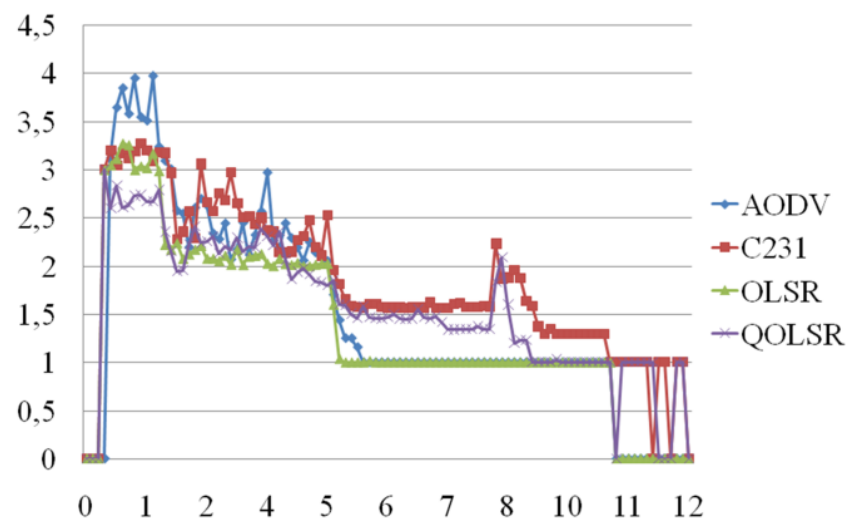

Application Traffic received

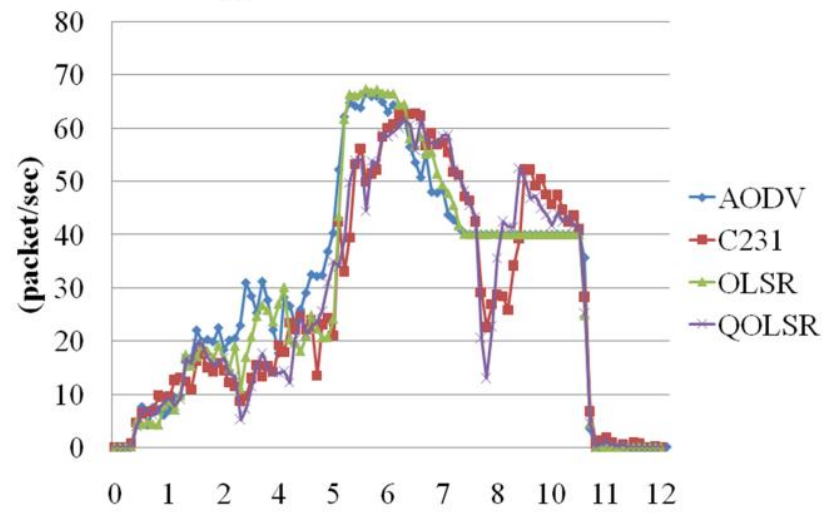

Application End-to-end delay

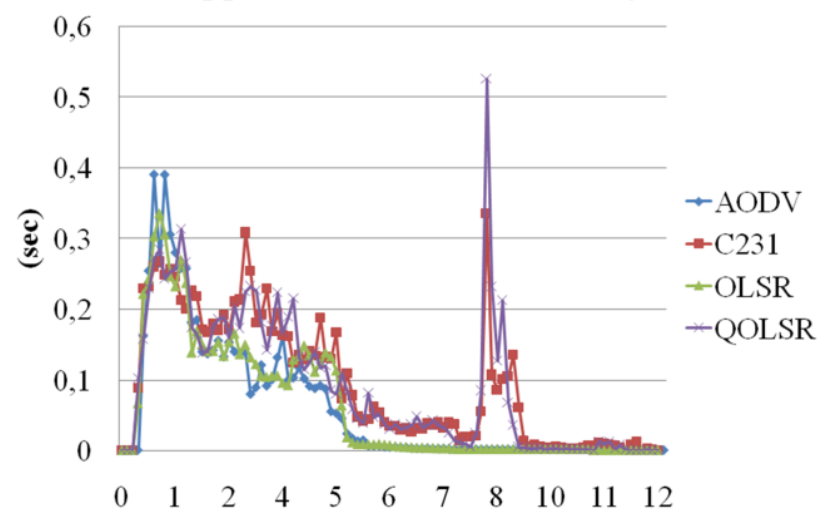

Fig. 8. Hop count, delivery, end-to-end delay for unicast traffic (scenario 4)

3) Results for scenarios 5 and $6:$ a voice application
IPHop count

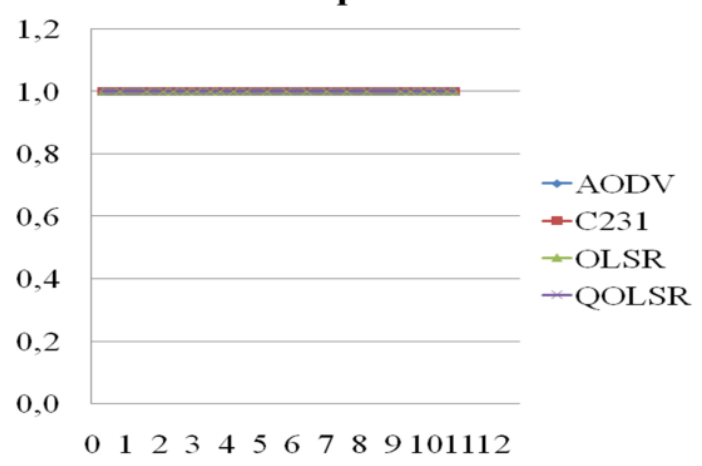

Voice end-to-end delay (1-hop)

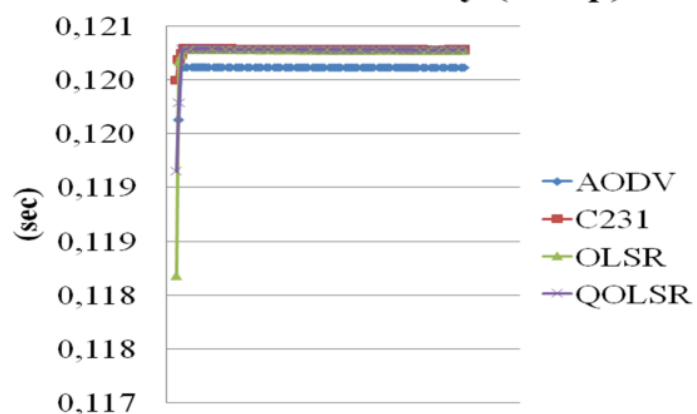

0123456789101112

Voice Mean Opinion Score value (1-hop)

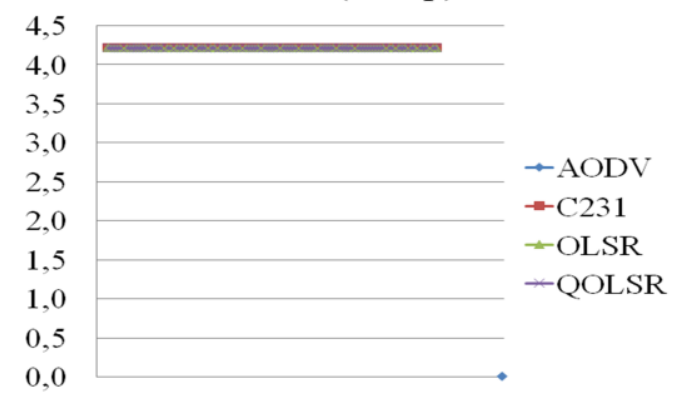

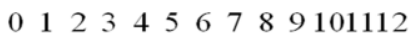

\section{Voice delivery (1-hop)}

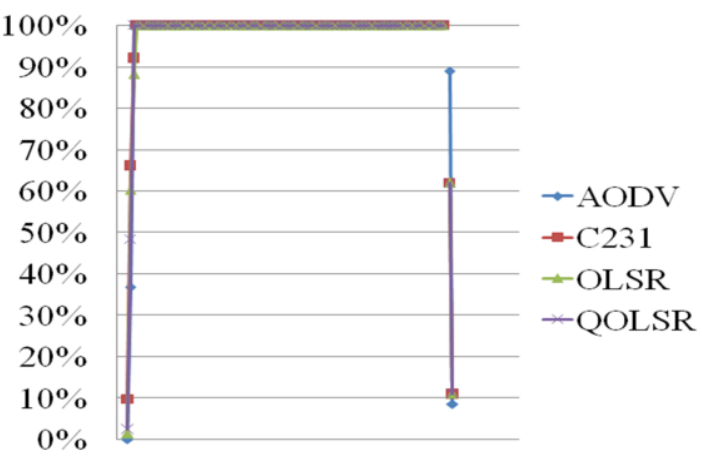

0123456789101112

Fig. 9. Hop count, end-to-end delay, MOS, delivery for voice (scenario 5) 


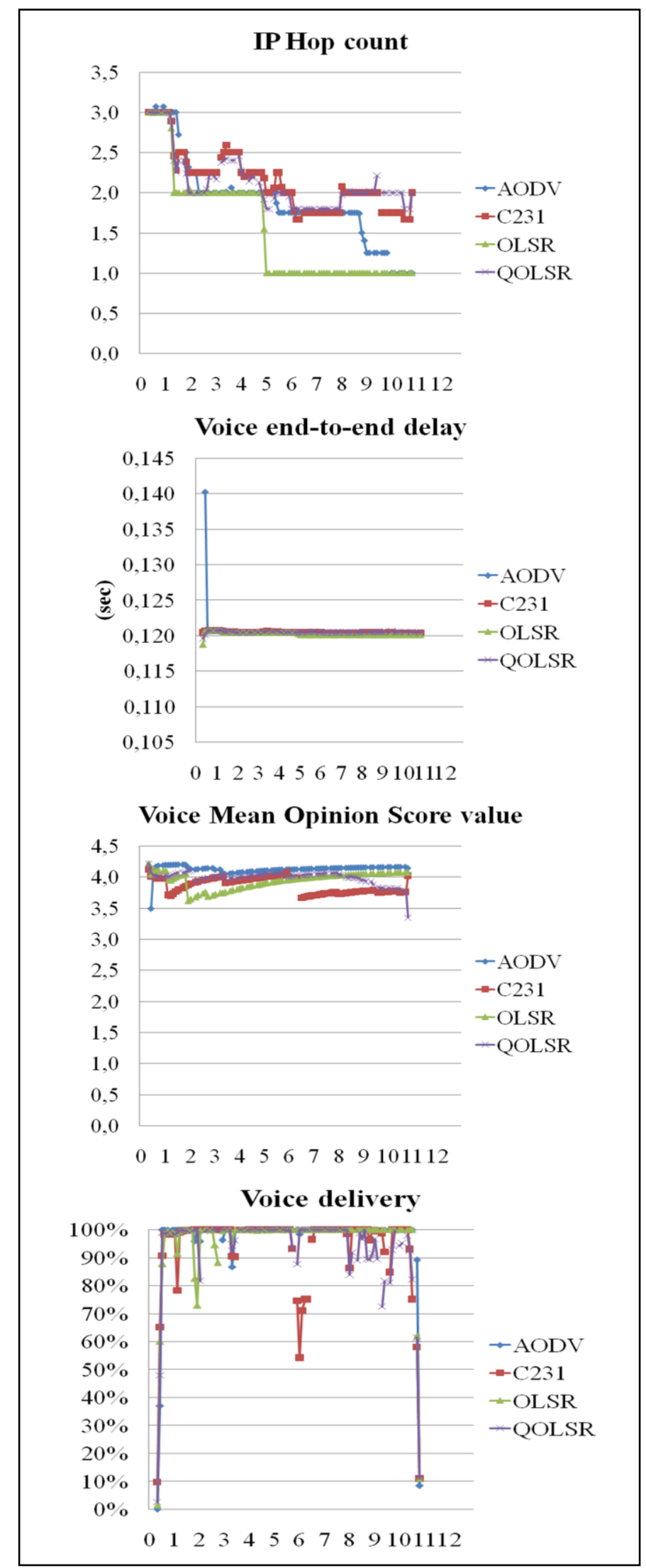

Fig. 10. Hop count, end-to-end delay, MOS, delivery for voice (scenario 6)
As shown on Fig. 9, voice communications operate perfectly 1-hop whatever the routing protocol, and despite the mobility of the vehicles. The delivery is $100 \%$, the delay is inferior to QoS delay constraint for voice $(150 \mathrm{~ms})$, and the mean opinion score (MOS) value superior to 4 indicates good communication quality comparable to GSM. When voice session is operated by 2-hop communicating pairs or farther (Fig. 10), delivery may fall to $80 \%$ for every routing protocols, even $60 \%$ for $\mathrm{C} 231$.

The MOS value still indicates good communication quality, but also clearly degradations when losses occur. AODV provides the best performance every time, while OLSR has the worst until the communication is 1-hop again. QOLSR and C231 operate better with this application with QoS requirements, QOLSR being the more efficient. The good results obtained with AODV demonstrate that $802.11 \mathrm{p}$ can offer a good support for voice application up to 3-hop communicating pairs provided that the related flow is the sole.

4) Results for scenarios 7 and 8 : videoconferencing

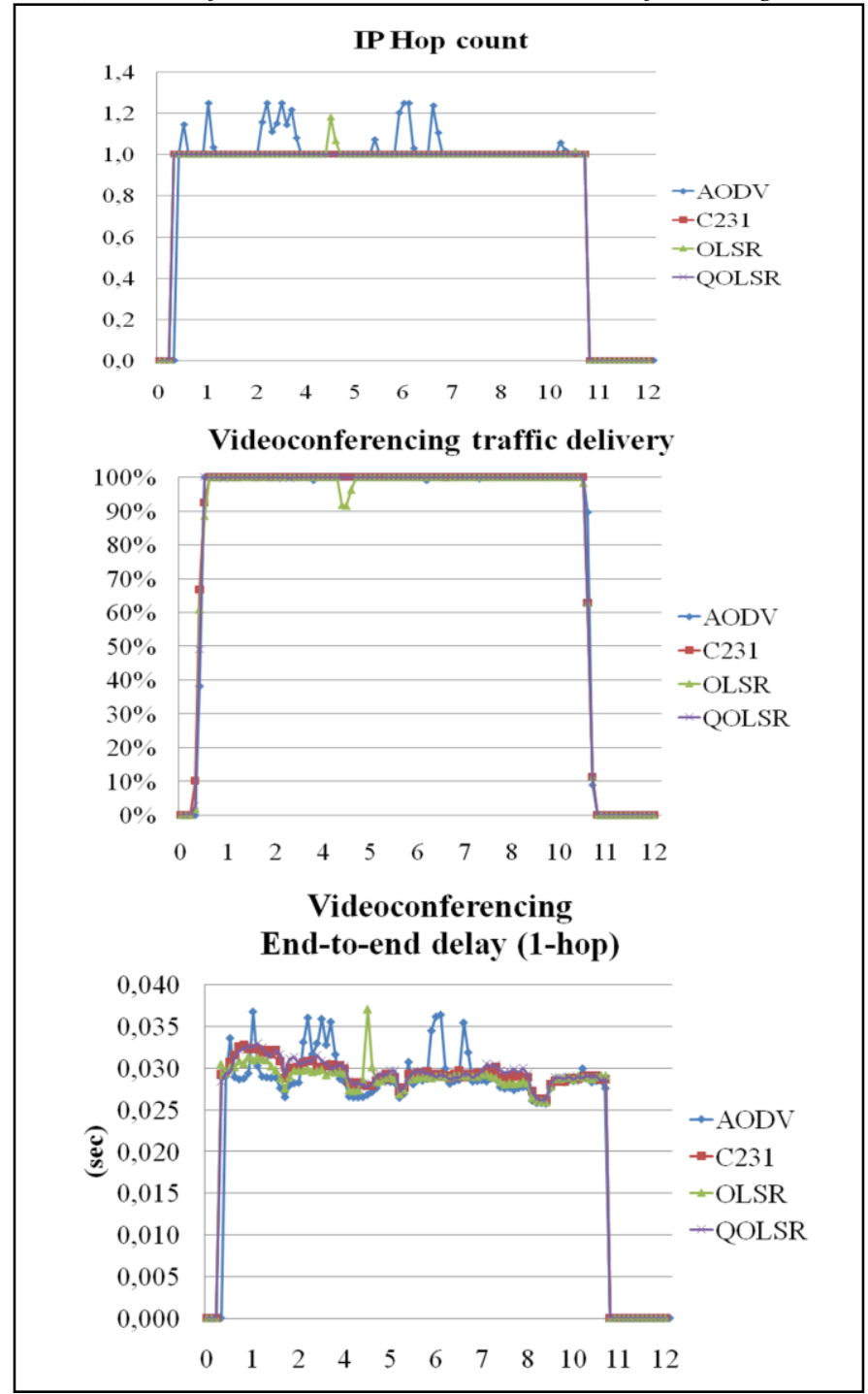

Fig. 11. Hop count, delivery, and delay for videoconferencing (scenario 7) 


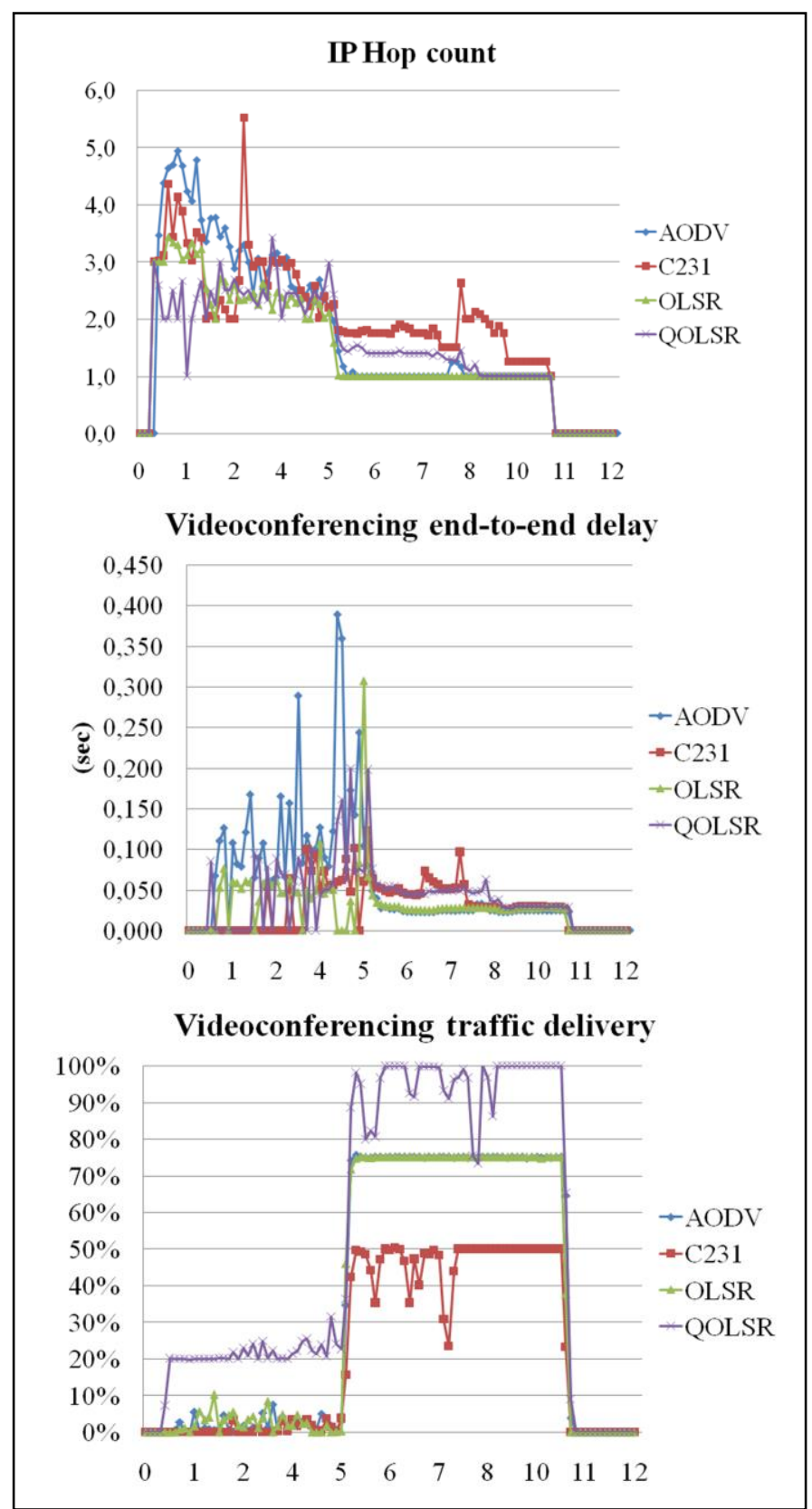

Fig. 12. Hop count, delivery, and delay for videoconferencing (scenario 8)

Videoconferencing application operates correctly when the communicating nodes are 1-hop away even under mobility conditions and for any of the routing protocols (Fig. 11). When multi-hop communications are necessary between the source and the destination, videoconferencing performance decreases dramatically (Fig. 12). These evaluations confirm the results in [4]: above 3-hop, the delivery of videoconferencing packets falls to $20 \%$ with OLSR and QOLSR, but also with AODV and C231. Despite additional routing traffic introduced by QOLSR, this variant still obtains higher packet delivery for videoconferencing than OLSR. AODV obtains the same packet delivery as OLSR, but due to longer routes AODV causes higher delays at the limit of videoconferencing threshold ( 300 ms) and very bad packet delay variation (above the limit of 50 $\mathrm{ms}$ for most of the packets). The strategy implemented in C231 based only on a single metric (i.e. received power) favors longer routes, thus degrading performance more than with other protocols. Combining three metrics which values are frequently updated as in QOLSR seem to be a better indicator for a good selection of relaying nodes for videoconferencing traffic. Indeed, QOLSR achieves the best performance despite the additional routing traffic necessary to its functioning.

\section{5) Results for scenarios 9: concurrent traffics}

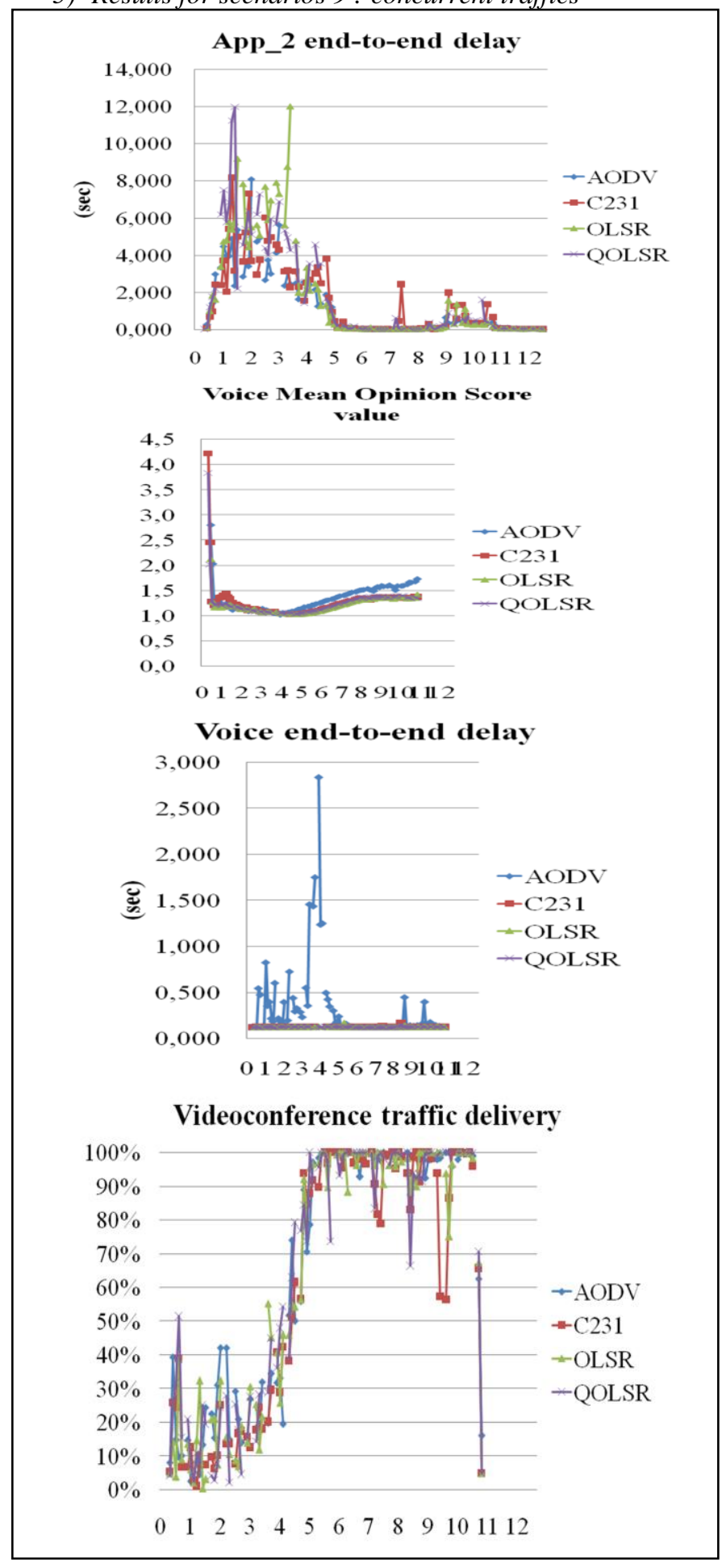

Fig. 13. Summary of results for the scenario 9 : concurrent traffics 
As scenario 9 was described in section IV.A, three communicating pairs exchange simultaneously a different application each: one pair exchanges unicast traffic, another voice and the other videoconferencing. The end-to-end delay for the unicast traffic related to the safety application (App_2 described in section III.E) increases above the seconds, out of the bounds for real-time application constraints. Voice application is affected completely, falling to very bad quality (mean opinion score value) even in 1-hop case despite a relative correct packet delivery. The routing protocols have almost no real determining effect on the performance, and only AODV behaves slightly differently by reaching very high delays for voice application. Videoconferencing delivery is still good only when the communicating nodes are 1-hop away, but the related delays are slightly higher compared to scenarios 7 and 8 where there were no concurrent traffic. It seems that it should be avoided to operate several critical or QoS constrained applications in the same time and in the same group of vehicles. Thus, in absence of infrastructure, there is a need for distributed coordination between the nodes in order to operate admission control to ensure that a only a single critical traffic occur. These results confirm the conclusions of the evaluations performed in [2]. Indeed, in presence of different types of traffic with different priority levels, $802.11 \mathrm{p}$ does not yet achieve good delivery for all of them.

6) Scalability of the wireless LAN in presence of traffic

The results presented in Fig. 14 represent respectively the wireless LAN state when only one application is running (first row: the results are the average over scenarios $1,3,4,5,6,7$, 8 ), when three applications are running (second row: the results come from scenario 9) and when a full mesh traffic occurs between every pairs in the network (third row: the results come from scenario 2 where 50 broadcast traffic flows were sent simultaneously). When reasonable traffic is sent (one or three applications running), AODV realizes a throughput close to the load. As a reactive routing protocol, AODV tries to find enough resources to fulfill the demand. OLSR and its variants tend to ensure half more and even double throughput values as compared to the load. Proactive routing protocols try to gather most resources possible in order to be ready to fulfill any demand upon request. In the full mesh traffic case, AODV throughput and delays dramatically increase when OLSR and its variants scale better. Designed for dense networks, OLSR optimizes broadcast using multipoint relaying techniques.

\section{CONCLUSION}

In this work, the performance evaluation of several applications that could be provided as services to vehicles over ad hoc vehicle-to-vehicle communications has been presented. The simulation evaluation have been performed using Riverbed (OPNET) Modeler on a network topology made of 50 nodes equipped with $802.11 \mathrm{p}$ technology and following realistic trajectories in the streets of Paris at regular and authorized speeds. The results show that almost all application types are very well supported provided that the source and the destination have a direct link. Particularly, it has been shown that introducing supplementary hops in a communication has more effect on end-to-end delays and loss rates than having more packets, packets of higher size, or even higher mobility. It has been observed that when several types of traffic are sent simultaneously in the network, those having stringent QoS requirements undergo higher degradation, especially voice application. Another result of this study is the following. Ad hoc reactive routing protocols degrade performance by increasing the delays, whereas proactive ones introduce the same counter performance by increasing the network load with their routing traffic. Those latter, especially OLSR and its variants, are more efficient for broadcast traffic, while AODV allows better performance for unicast best effort traffic.

Whatever the routing protocol type adopted, the best performance seems to be obtained by maintaining small group of nodes reaching each other through at most two-hop routes. It will be particularly relevant, when the nodes can organize themselves, to avoid introducing other traffic while a session of an application with stringent QoS requirements is already running. Future study will investigate such self-organizing mechanisms, and try to lightening routing traffic induced by QoS signaling by improving the solutions such as the one evaluated in this work as C231 protocol.

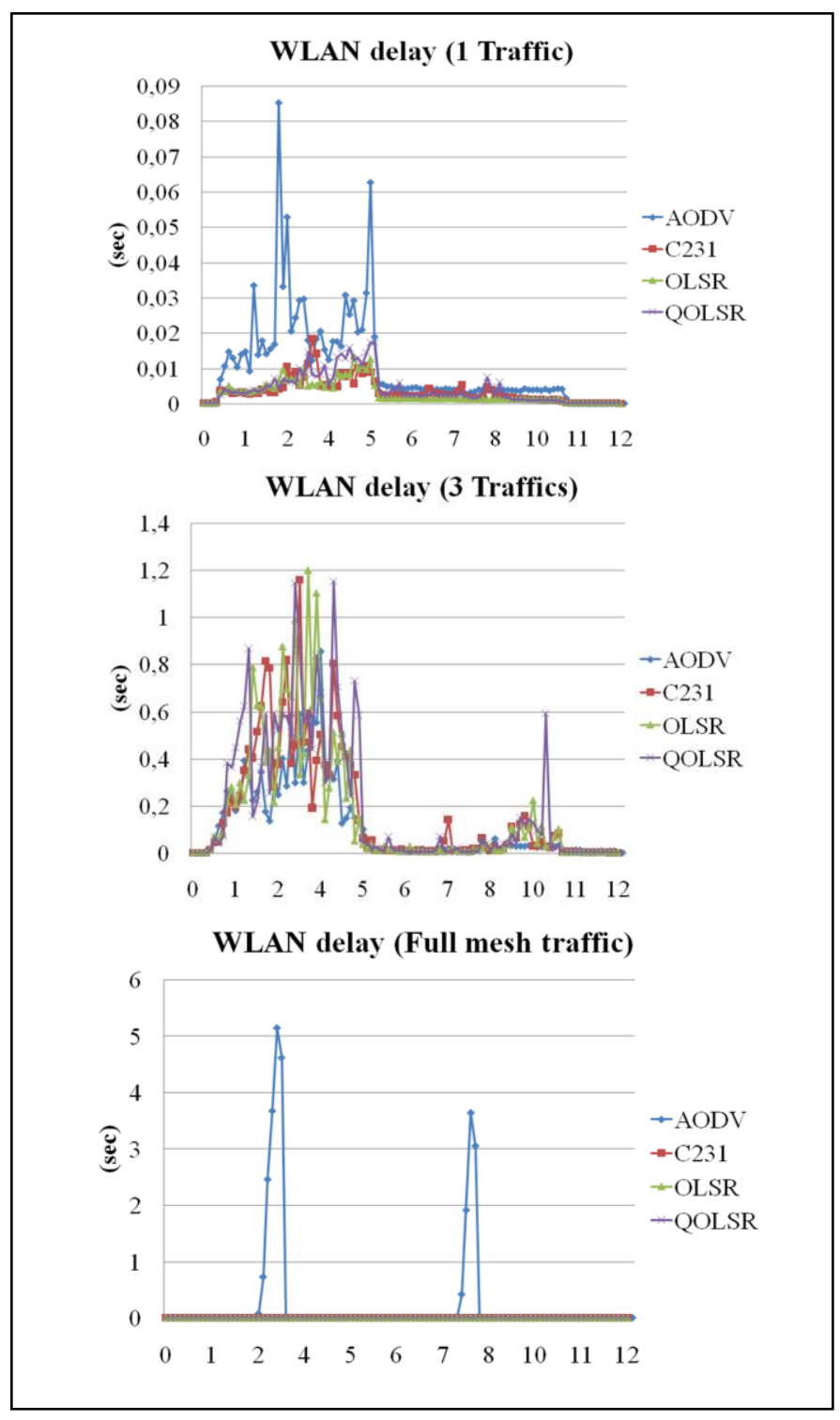

Fig. 14. Wireless LAN state evlolutions in presence of traffic 


\section{ACKNOWLEDGMENT}

The authors gratefully acknowledge the support of the CPER ELSAT 2020, the Nord-Pas-de-Calais Region, the European Community and the French National Center for Scientific Research.

\section{REFERENCES}

[1] M. Gerla, "Electric and autonomous vehicles in smart cities: impact on energy, transport and communication infrastructures", IEEE MMTC COMSOC E-Letter, vol. 10, $\mathrm{n}^{\circ} 3$, may 2015.

[2] Ning sun, "Performance study of IEEE802.11P for vehicle to vehicle communications using OPNET", a thesis presented in partial fulfillment of the requirements for the degree of Master of engineering in Telecommunications and network; Massy University, Auckland, New Zeland, November 2011.

[3] V.D. Khairnar,Dr. KetanKotecha , "Performance of Vehicle-to-Vehicle Communication using IEEE 802.11p in VehicularAd-hoc Network Environment", International Journal of Network Security \& Its Applications (IJNSA), Vol.5, No.2, March 2013.

[4] Bilstrup K.,Uhlemann E., Strom E.G., Bilstrup U., "Evaluation of the IEEE 802.11p MAC Method for Vehicle-to-Vehicle Communication", Vehicular Technology Conference, 2008. VTC 2008-Fall. IEEE 68th, p.1-5, September 21-24, 2008.

[5] Stephan Eichler, "Performance Evaluation of the IEEE 802.11p WAVE Communication Standard", Proceedings of the 66th IEEE Vehicular Technology Conference, VTC Fall 2007, Baltimore, MD, USA., 30 September - 3 October 2007.

[6] B. E. Bilgin and V. C. Gungor, "Performance Comparison of IEEE 802.11p and IEEE 802.11b for Vehicle-to-Vehicle Communications in Highway, Rural, and Urban Areas", International Journal of Vehicular Technology, Vol. 2013, Article ID 971684, 10 pages, http://dx.doi.org/10.1155/2013/971684, 2013.

[7] Duddalwar P., Deshmukh A., Dorle SS., "A Comparative Study of Routing Protocol in Vehicular Ad Hoc Network", International Journal of Emerging Technology and Advanced Engineering, Volume 2, Issue 3, pp. 71-76, March 2012

[8] Felipe Domingos da Cunha, AzzedineBoukerche, Leandro Villas, Aline CarneiroViana, Antonio A. F. Loureiro, "Data Communication in VANETs: A Survey, Challenges and Applications", Research Report RR-8498, INRIA Saclay. 2014. HAL Id: hal-00981126, https://hal.inria.fr/hal-00981126v3

[9] Dua, A., Kumar, N., and , S. " A systematic review on routing protocols for Vehicular Ad Hoc Networks", Vehicular Communications (Elsevier) Journal, Volume 1 (1), 2014, Pages 33-52

[10] Johnson, D., Hu, Y. and Maltz, D. (2007), "The dynamic Source Routing Protocol (DSR) for Mobile Ad Hoc Networks for IPv4", IETF, RFC 4728 (2007)
[11] Perkins, C., Belding-Royer, E. and Das, S. (2003), "Ad hoc On-Demand Distance Vector(AODV) Routing”, IETF, RFC 3561 (2003)

[12] Clausen, T., and Jacquet, P. (2003), "OptimizedLink State Routing Protocol (OLSR)", IETF, RFC 3626 (2003)

[13] Ogier, R., Templin, F. and Lewis, M. (2004), "Topology Dissemination Based on Reverse-Path Forwarding (TBRPF)", IETF, RFC 3684 (2004)

[14] Roy, A. and Chandra, M. (2010), "Extensions to OSPF to Support Mobile Ad HocNetworking", IETF, RFC 5820 (2010)

[15] Sondi P., Ganstou D. and Lecomte S. (2013), « Design Guidelines for Quality of Service Support in Optimized Link State Routing-Based Mobile Ad Hoc Networks », Ad Hoc Networks (Elsevier) Journal, Volume 11 issue 1, pp. 298-323, 2013

[16] Sondi P., Ganstou D. and Lecomte S. «A Multiple-Metric QoS-Aware Implementation of the Optimized Link State Routing Protocol»,Int. J. Communication Networks and Distributed Systems, Vol. 12, № 4, 2014

[17] Euisin Lee; Eun-Kyu Lee; Gerla, M.; Oh, S.Y., "Vehicular cloud networking: architecture and design principles," Communications Magazine, IEEE , vol.52, no.2, pp.148,155, February 2014

[18] G. Amoussou, B. L. Agba, Z. Dziong, M. Kadoch, F. Gagnon, "Performances Analysis of mobile ad hoc routing protocols under realistic mobility and power models", Session 1542, OPNETWORK'06 Washington D.C., August 28 - September 1, 2006.

[19] Fan Ya-qin; Fan Wen-yong; Wang Lin-zhu, "OPNET-based Network of MANET Routing Protocols DSR Computer Simulation", International Conference on Information Engineering (ICIE), 2010 WASE

[20] Vidhale, B.; Dorle, S.S." Performance Analysis of Routing Protocols in Realistic Environment for Vehicular Ad Hoc Networks", 21st International Conference on Systems Engineering (ICSEng), 2011, Pages: 267 - 272, DOI: 10.1109/ICSEng.2011.55.

[21] Tapan K. Sarkar, Zhong Ji, Kyungjung Kim, AbdellatifMedour "A Survey of Various Propagation Models for Mobile Communication" IEEE Antennas and Propagation Magazine, Vol. 45, No. 3, June 2003.

[22] R. Mardeni and K. F. Kwan "optimization of hata propagation prediction model in suburban area in Malaysia" Progress in Electromagnetics Research C, Vol. 13, 106, 2010.

[23] S. IbenJellal, O. Cohin, S. Baranowski, U. Biaou, M. Bocquet, A. Rivencq, " Experimental analysis of Zigbee RF signal performances for railway application:Study on a laboratory reduced scale train", IEEE International Conference on Advanced Logistics and Transport, 2015

[24] Vishal D. Nimavat, G. R. Kulkarni , "Simulation and Performance Evaluation of GSM propagation Channel under the Urban, Suburban and Rural Environments", 2012 International Conference on Communication, Information \& Computing Technology (ICCICT), Oct. 19-20, Mumbai, India, 2012 